\title{
MARKET STRUCTURE AND RESOURCE EXTRACTION UNDER UNCERTAINTY*
}

\author{
Joseph E. Stiglitz \\ Princeton University, Princeton, New Jersey, USA
}

Partha Dasgupta

London School of Economics, London, England

\begin{abstract}
This paper compares the rate of extraction of a natural resource under alternative market structures when there is uncertainty about the date of discovery of a substitute (or about the date of discovery of a new deposit). The analysis shows that imperfectly competitive market structures are excessively conservationist: for any value of the initial stock of the natural resource, the price is higher than in competitive equilibrium (and therefore higher than the socially optimal level). But markets with limited competition may have a higher price than markets with pure monopoly (the same monopolist controlling the natural resource and its substitute). In particular, we find that the highest prices are associated with (Nash quantity setting) duopolists; the next highest prices occur in markets in which the monopolist controls the resource but the substitute is competitively produced; the pure monopolist sets his price lower than this, while the market in which a monopolist controls the substitute but the resource is competitvely owned is still lower.

One of the strategies commonly proposed for responding to the OPEC cartel is for the consuming nations to develop substitutes which they could then ensure would be competitively supplied. Our analysis suggests that the response of the resource monopolist to this change in market structure would be to raise his prices, not to lower them.
\end{abstract}

\section{Introduction}

In this paper we compare the rate of extraction of a natural resource when there is uncertainty about the date of discovery of a substitute ${ }^{1}$ under alternative market structures.

\footnotetext{
* This paper is a revised version of results originally reported in Dasgupta \& Stiglitz, "Uncertainty and rates of extraction under alternative institutional arrangements", IMSSS Technical Report No. 179, Stanford, 1976. An earlier draft of this paper was completed while Stiglitz was Oskar Morgenstern Distinguished Research Fellow at Mathematica and visiting professor at the Institute for Advanced Study. Financial support from the National Science Foundation is gratefully acknowledged.

1 The effect of uncertainty about the date of discovery of a new deposit may be analyzed in a very similar manner.
}

Scand. J. of Economics 1981 
In an earlier paper, we compare the rate of extraction (and the date of innovation of the substitute) after the discovery occurs; see Stiglitz \& Dasgupta (forthcoming). In this paper, we are concerned with the rate of extraction prior to the discovery of the substitute.

In our earlier paper, we were able to show how, under each of a variety of market structures, the price at the date of invention could be determined; for instance, the price in monopoly, $p^{m}(S)$, for every value of the stock, $S$, of the resource, was shown always to be greater than the price in competition (or the socially optimal price), for the corresponding value of the stock, $p^{s}(S)$. The fact that once the new substitute has been developed the price will be higher at each value of $S$ naturally leads to the conjecture that prior to the invention, since the invention reduces the value of the natural resource, the resource will be used up less quickly, i.e. prior to the invention, the price will be higher, at each value of the stock.

This conjecture turns out to be correct for the particular comparison we have just made- between monopoly and pure competition. Thus, we are able to extend the result of our earlier analysis showing that monopolies have a bias to excessive conservationism to the period prior to the invention having occurred.

The conjecture, however, is not true generally: one cannot infer from the comparison of post-invention prices what pre-invention extraction rates will be.

In our earlier analysis, we showed that in some models of limited competition ${ }^{1}$ the equilibrium did not lie between the polar cases of pure competition and pure monopoly. For these same models of limited competition, we show again that the rate of extraction need not be between those for the two "polar" cases of pure monopoly and pure competition. Moreover, even if the price immediately after the invention is less than in the pure monopoly, prior to the invention the market may be more conservationist even than pure monopoly.

The reason for these unexpected results is simple. A monopolist owning a natural resource in deciding his optimal extraction policy is concerned with the marginal revenue of an extra unit of the resource after the invention has been discovered. The relationship between marginal revenues and prices may differ markedly in different market structures. In particular, although the post invention price may be lower when the monopoly resource owner faces a competitive substitute than when the monopolist also controls the substitute, his marginal revenue may be higher, and it is this which leads him in the preinvention period to charge higher prices for the natural resource.

The actions which the owners of resources can take prior to the discovery We use the term limited competition to describe market structures in which there is
more than one firm but at least one firm can affect market price significantly.

Scand. J. of Economics 1981 
of the substitute have important implications for the timing of innovation ${ }^{1}$ and, where the discovery date of the invention itself is affected, to some extent, by expenditures on $R \& D$, on the level of those expenditures and the development of substitutes; in particular, our analysis suggests that certain forms of limited competition may be associated with particularly long lags in innovation. These questions are discussed in greater detail in Dasgupta, Gilbert \& Stiglitz (1980a, 1980b), Stiglitz, Gilbert \& Dasgupta (1978) and Dasgupta \& Stiglitz (1980).

We examine in this paper five market structures:

(a) pure competition, or, equivalently, the socially optimal allocation of resources;

(b) pure monopoly, where the same firm owns both the resource and its substitute;

(c) competitive resource market, monopoly control of substitute; this is perhaps the most relevant case, where the government grants a patent to the developer of the substitute;

(d) competitive substitute, monopoly resource; if one views OPEC as acting as a collusive cartel, and the consuming governments as developing a substitute, the technology for producing which they will make freely available, this is an appropriate model for analyzing the oil market;

(e) one firm controls the natural resource, and another firm controls the substitute (duopoly).

\section{The Basic Arbitrage Equation for a Competitively Owned Resource}

Let $S_{t}$ denote the stock of an exhaustible resource at $t$. We take it that the resource is owned competitively. Let $p_{t}$ denote the spot price of the pre-extracted resource at $t$ and let $r_{t}$ denote the competitive market rate of interest. Consider a short interval of time $(t, t+\theta)$. Suppose that the probability that some specific event will occur during this interval is $\lambda_{t} \theta$. If the event does occur, the competitive price will be $\hat{p}_{t}$. We refer to $\hat{p}_{t}$ as the fall-back price of the resource at $t$. Presumably this fall-back price will depend on $S_{t}$, and the specific nature of the event. Thus, we write $\hat{p}_{t}=\hat{p}_{t}\left(S_{t}\right)$. If the event does not occur during this interval the price at $t+\theta$ will be $p_{t+\theta}=p_{t}+d p_{t}$. If speculators are risk neutral in this economy then in dynamic equilibrium one will have

$\lambda_{t} \theta \hat{p}_{t}\left(S_{t}\right)+\left(1-\lambda_{t} \theta\right)\left(p_{t}+d p_{t}\right)=\left(1+r_{t} \theta\right) p_{t}$,

which, on taking limits as $\theta \rightarrow 0$ yields the basic arbitrage condition

$\frac{\dot{p}_{t}}{p_{t}}=r_{t}+\lambda_{t}\left[1-\frac{\hat{p}\left(S_{t}\right)}{p_{t}}\right]$.

1 We follow the usual convention of defining the date of invention as the date at which it becomes possible to produce the substitute, the date of innovation as the date at which the substitute actually gets produced.

Scand. J. of Economics 1981 
Equation (2.2) is, of course, a very general one; it will for instance be valid even when one contemplates an entire sequence of possible events over time. It represents the equilibrium condition at $t$, so that $\lambda_{t}$ denotes the probability density that a specific event occurs at $t$ conditional on it not having occurred earlier.

Certain special cases of (2.2) may now be mentioned. If either $\lambda_{t}=0$ (i.e., there is no chance that the event will occur at $t$ ) or if $\hat{p}\left(S_{t}\right)=p_{t}$ (i.e., the event is a trivial one and has no bearing on the market for the resource), then $\dot{p}_{t} / p_{t}=r_{t}$. It is this special case that has been analyzed at length in the earlier literature. ${ }^{1}$ Given that we have not specified the precise nature of the event and, consequently, that we do not know what the fall-back price is, it may appear as though equation (2.2) is far too general to enable one to obtain any specific insight into the rate of price change of the resource. But in fact, for one large class of cases the equation provides a useful inequality. Suppose the event contemplated at $t$ is a "beneficial" one (e.g., the discovery of a new reserve of the resource or the invention of a substitute product). Then clearly $\dot{p}\left(S_{t}\right) \leqslant p_{t}$. In such a situation equation (2.2) implies that

$r_{t} \leqslant \frac{\dot{p}_{t}}{p_{t}} \leqslant r_{t}+\lambda_{t}$

Thus under competitive equilibrium the possibility of a beneficial event occurring at $t$ carries with it the implication that the rate of price rise of an exhaustible resource will be in excess of the rate of interest at $t$. But the rate of price rise will be bounded above by the sum of the interest rate and the probability density of the occurrence of the event at $t$ conditional on its not having occurred prior to $t$. It is only when $\hat{p}\left(S_{t}\right)=0$ (i.e., the event renders the existing stock worthless), that

$\dot{p}_{t} / p_{t}=r_{t}+\lambda_{t}{ }^{2}$

As we have remarked earlier, in order to give more structure to the problem we shall visualize the possibility of the occurrence of a single event-the discovery of a substitute product that can be produced at unit cost $\bar{p} . \bar{p}$ is known with certainty but the date of occurrence of this invention is random. In other words, after the invention occurs (if it does occur, that is) there is no remaining uncertainty for the economy in question. ${ }^{3}$ Given this, the information tree for our model is simple and is described, for discrete time, in Fig. 1. The world line $0 A$ represents the non-occurrence of this single event. 1 See, for instance, Hotelling (1931), Herfindahl (1967), Solow (1974a), Stiglitz (1977) and
Sweeney (1974).

2 This is the special case analyzed by Dasgupta \& Heal (1977).

It will become evident from the discussion below that if instead we considered the situa. tion where at some date $t$ it becomes known that the invention will occur at some subsequent date $t+T$, then there will be a discontinuity in the price of the resource at $t$, the date at which the information becomes available. There may be a further discontinuity
in the price at $t+T$. 


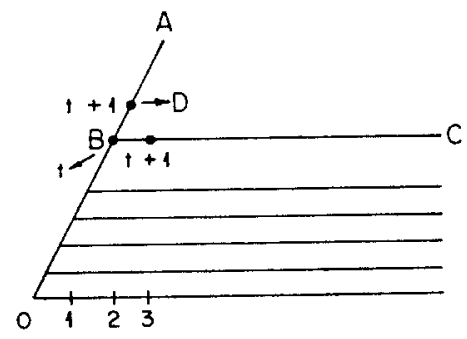

Fig. 1

The nodal point $B$ represents the state of the world at $t$ conditional on the discovery not having been made prior to $t$. At this point $\lambda_{t}$ is the probability that the economy will follow the branch $B C$ and $\left(1-\lambda_{t}\right)$ is the probability that it will move to $D$.

Now equation (2.2) has been arrived at from the postulate of risk neutral speculators. It is clear though that we could have as well arrived at it by postulating the existence of a complete set of contingent futures markets, thereby removing all uncertainty from the resource owners, provided that the market as a whole acts in a risk neutral manner to this particular risk (e.g., because its consequences are small relative to national income). Using continuous time representation and assuming $\lambda$ constant and $\vec{p}=0$ (i.e., the process is a Poisson one) $p_{t} e^{-(r+\lambda) t}$ would denote the price to be paid at the point 0 for the delivery of a unit of the resource at $B .^{1}$ It is also clear that equation (2.2) would describe the behavior of the imputed price of the resource, were both the resource and the substitute to be socially managed. This last is of course what basic welfare economics would imply.

In this paper our focus of attention will be on the rate of extraction and the fall-back price $\hat{p}\left(S_{t}\right)$, both of which will be endogenous for our system. Consequently, we take it that $r_{t}$ is given exogenously and, for simplicity, that $r_{t}=r$ (a constant, $>0$ ). We shall also assume here that $\lambda_{t}$ is uninfluenced by policy. ${ }^{2}$ Again, for simplicity, we shall assume that $\lambda_{t}=\lambda$ (a constant).

\section{Analysis of Socially Optimal Patterns of Allocation}

We can use the arbitrage equation to solve for the equilibrium rate of extraction in the period prior to the invention occurring, provided we know the function $\hat{p}(S)$, giving the fall-back price as a function of the stock. We simplify as before and assume $r$ and $\lambda$ are independent of time. Our market is described then by the price differential equation,

\footnotetext{
1 We shall naturally calculate the entire set of contingent prices after articulating the model in detail.

2 In Dasgupta \& Stiglitz (1980 $a$ and $b$ ), Dasgupta, Gilbert \& Stiglitz (1980a and $b$ ) and Stiglitz, Gilbert \& Dasgupta (1978) the rate of technical progress is taken to be endogenous.
}

Scand. J. of Economics 1981 
$\dot{p}=(r+\lambda) p-\lambda \dot{p}(S)$

and by the extraction equation

$\zeta=-D(p)$,

where $D(p)$ is the demand for the resource as a function of the price. We postulate that $D(p)>0$ for all $p$. For simplicity we focus on the case of constant elasticity demand functions ${ }^{1}$

$Q=p^{-\varepsilon}, \varepsilon>1$.

In addition to these two differential equations, we need two boundary conditions: one gives the initial stock, $S_{0}$, and ${ }^{2}$ the other says that, if the invention never occurs, we only use up the resource asymptotically.

$\lim _{t \rightarrow \infty} S=0, \quad S(t)>0$ for all finite $t$.

In Fig. 2, we have presented the phase diagram for this market. Clearly, $\dot{p}=0 \quad$ along the curve

$p=\frac{\lambda \hat{p}(S)}{r+\lambda}$

and above the $\dot{p}=0$ curve, $\dot{p}>0$.

In Stiglitz and Dasgupta (forthcoming) we establish the not surprising result that the fall back price is a declining function of the stock:

$\hat{p}^{\prime}(S)<0$.

Thus, the $\dot{p}=0$ locus is downward sloping.

Since we postulate that $D(p)>0$ for all $p$, the phase diagram appears as drawn. Working with the boundary condition (3.3) is slightly awkward; it is easier if we "guess" a value of $p_{0}$.

We can easily solve (3.1) and (3.2) for any given initial values of $p_{0}, S_{0}$. The solutions as a function of $p_{0}$ for given $S_{0}$ are illustrated in Fig. 3. The critical property of the solution ${ }^{3}$

\footnotetext{
1 We choose this class of functions not only for the analytical simplicity, but also because we know that in the simplest models, it is precisely this class of functions which gives rise to no bias in the monopoly rate of resource extraction; Stiglitz (1977). The restriction to elasticities greater than unity is, of course, necessary in the analysis of monopolistic market
structures.

2 This is the familiar transversality condition of optimal growth theory. Clearly, if $\lim _{t \rightarrow \infty} S>0$, the trajectory is inefficient, while if $S=0$ at $t=T<\infty$, the "virtual" price, given our constant elasticity demand curve, is infinite for all $t>T$, and hence again the
path cannot be efficient.

It follows directly from observing that

$\frac{\partial \dot{p}}{\partial p}>0 ; \quad \frac{\partial \dot{p}}{\partial S}=-\lambda \hat{p}^{\prime}>0$.

$\frac{\partial \dot{S}}{\partial p}=-D^{\prime}>0$
}

Scand. J. of Economics 1981 
$324 J$. E. Stiglitz and P. Dasgupta

$\left\{p\left(t ; S_{0}, p_{0}\right), S\left(t ; S_{0}, p_{0}\right)\right\}$

is that

$\frac{\partial p}{\partial p_{0}}>0, \frac{\partial S}{\partial p_{0}}>0$, all $t, S_{0}$

at each $t$, price is a monotonically increasing function of initial price and the stock remaining is a monotonically increasing function of $p_{0}$.

Thus, for $p_{0}$ very large, $\lim _{t \rightarrow \infty} S_{t}>0,{ }^{1}$ while for $p_{0}$ very small, $S$ becomes 0 in finite time. There thus exists a critical value of $p_{0}$, denoted $p_{0}^{s}$, satisfying the

$\frac{\dot{p}}{p}=r+\lambda-\frac{\lambda \dot{p}}{p}<r+\lambda$,

i.e.

$p<p_{0} e^{(r+\lambda) t}$

$-\dot{S}>p_{0}^{-\varepsilon} e^{-(r+\lambda) \varepsilon t}$

$S_{t}<S_{0}-\frac{p_{0}^{-\varepsilon}\left(1-e^{-(r+\lambda) \varepsilon t}\right)}{(r+\lambda) \varepsilon}<0$

for

$p_{0}<\left[\frac{(r+\lambda) \varepsilon S_{0}}{1-e^{-(r+\lambda) \varepsilon t}}\right]^{-1 / \varepsilon}$

Similarly

$\frac{1}{\lambda} \frac{d \dot{p} / p}{d t}=-\frac{\hat{p}^{\prime} \dot{S}}{p}+\frac{\dot{p}}{p} \frac{\dot{p}}{p}$

It can be shown, as in Proposition 1 in Stiglitz \& Dasgupta (forthcoming), that in the postinvention phase,

$p=\hat{p} e^{r t}$

$\dot{S}=-D(p)$.

Hence

$\frac{d \hat{p}}{d S}=-\frac{\hat{p} r}{D}, \quad$ and

$\frac{1}{\lambda} \frac{d \dot{p} / p}{d t}=(r+\lambda) \frac{\hat{p}}{p}-\left(\frac{\hat{p}}{p}\right)^{2} \lambda-r \frac{\hat{p}}{p}>0$

Hence

$p>p_{0} e^{\left[r+\lambda-\lambda \hat{p}\left(S_{0}\right) / p_{0}\right] t}$

Hence

$\lim _{t \rightarrow \infty} S_{t}>S_{0}-\frac{p_{0}^{-\varepsilon}\left(1-e^{-\left(r+\lambda-\lambda \hat{p}\left(S_{0}\right) / p_{0}\right) \varepsilon t}\right)}{\left(r+\lambda-\hat{p}\left(S_{0}\right) / p_{0}\right) \varepsilon}>0$

for $p_{0}$ sufficiently large.

Scand. J. of Economics 1981 


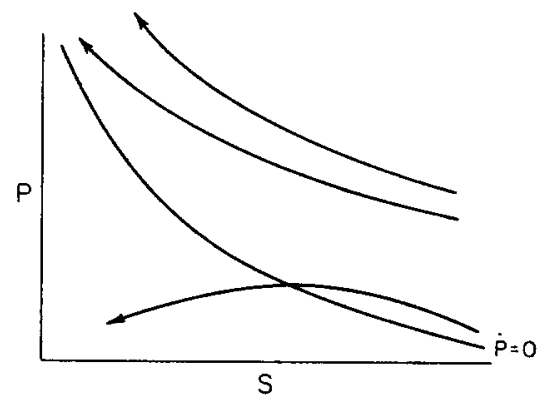

Fig. 2. Phase diagram for competitive economy.
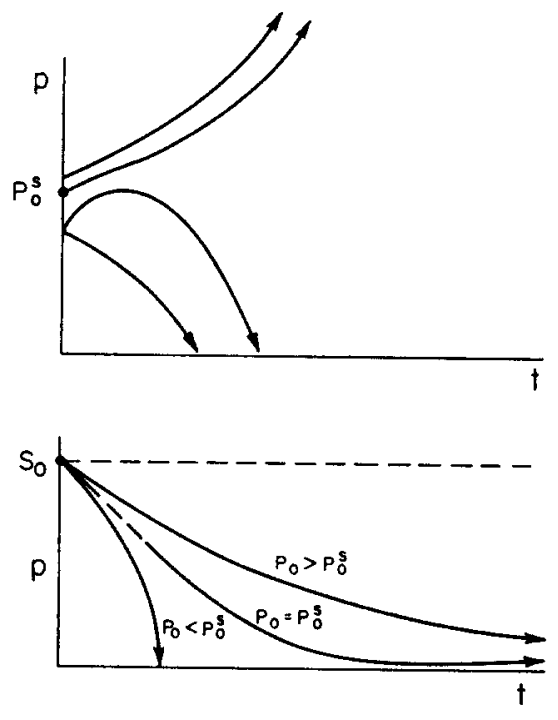

Price and Stock as a Function of Time

Fig. 3. Price and stock as a function of time

differential equations and the boundary conditions $S(0)=S_{0}$ and (3.3). It is also immediate that $d p_{0} / d S_{0}<0$.

We summarize the result as

Proposition 1. There exists a unique, optimal extraction policy satisfying (3.1)(3.3); the price of the resource increases monotonically over time at a rate less than $r+\lambda$; the price is a monotonically declining function of the size of the stock of the resource remaining:

$p^{s}=p^{s}(S) \frac{d p^{s}}{d S}<0$

Proposition 1 provides a basis for comparing extraction rates with and without uncertainty; our intent here, however, is not in that comparison 


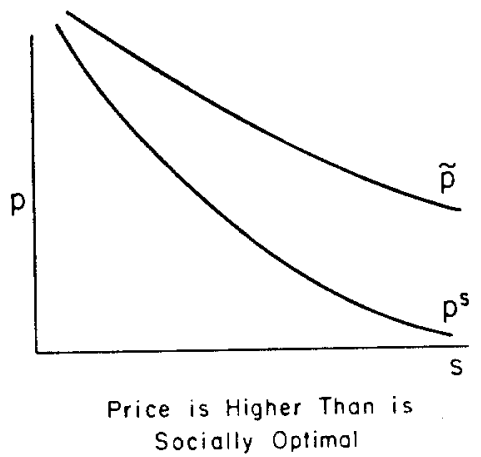

Fig. 4. Price is higher than is socially optimal.

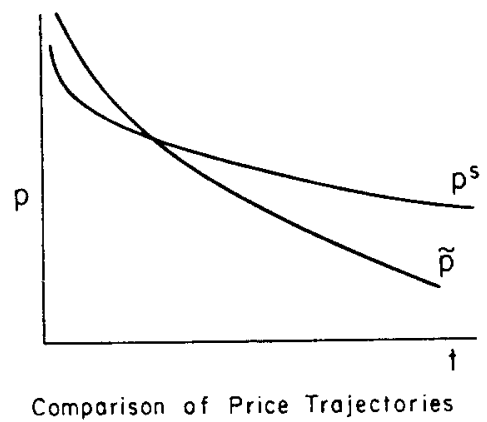

Fig. 5. Comparison of price trajectories.

but in a comparison of the patterns of extraction under alternative market structures; see Dasgupta-Stiglitz (1979).

\section{Innovation Patented}

The easiest comparison to make is between the socially optimal allocation and that where the substitute will be controlled by a single producer (e.g., as a result of a patent). This, of course, would typically be the case, except if the research was publicly funded; and the fact that the substitute is controlled by a single firm does not mean the economy is not competitive--in the conventional sense of that term-for there may have been competition in the research process, the patent holder being the winner in that competitive struggle, and there may be continuing competition for developing new, lower costs substitutes.

The analysis proceeds exactly as in the purely competitive (socially optimal) case, except the fall back price is now different. The fact that the market after the invention is not competitive leads the price after the invention to be higher than it is in the socially optimal allocation. Denoting by $\hat{\tilde{p}}$ the fall-back price Scand. J. of Economics 1981 
in this market structure, it is shown in Stiglitz \& Dasgupta (Proposition 3 b) that

$\hat{\tilde{p}}(S)>\hat{p}^{s}(S)$

To see what this implies for the pre-invention market, we need first to prove

Lemma 1. Consider the following two pairs of differential equations:

$$
\begin{aligned}
& \dot{x}=a x-b_{1}(y) \quad b_{1}^{\prime}<0, b_{1}>0, a>0 \\
& \dot{y}=-c(x) \quad c^{\prime}<0, c>0 \text { all } x>0 \\
& x(0)=x_{0}>0 \\
& \lim _{t \rightarrow \infty} y=0 . \\
& \dot{x}=a x-b_{2}(y) \quad b_{2}^{\prime}(y)<0, b_{2}>b_{1}>0 \\
& \dot{y}=-c(x) \\
& x(0)=x_{0}>0 \\
& \lim _{t \rightarrow \infty} y=0 .
\end{aligned}
$$

Let $x_{1}(t), y_{1}(t)$ be the solution to (4.2), $x_{2}(t), y_{2}(t)$ be the solution to (4.3) and let $x_{1}(y)$ and $x_{2}(y)$ be the solution in $(x, y)$ phase space. Then

$x_{1}(y)<x_{2}(y)$

for all $y$.

Proof. Assume $x_{1}(\tilde{y}) \geqslant x_{2}(\tilde{y})$ for some $\tilde{y}$. Let $y_{1}\left(t_{1}\right)=\tilde{y}, y_{2}\left(t_{2}\right)=\tilde{y}$. Then when $y=\tilde{y}, \dot{x}_{1}>\dot{x}_{2}, \dot{y}_{1} \geqslant \dot{y}_{2}$. Since

$\frac{\partial \dot{x}}{\partial x}=a>0, \quad \frac{\partial \dot{x}}{\partial y}=-b^{\prime}>0$,

$x_{1}\left(t+t_{1}\right)>x_{2}\left(t+t_{2}\right), y_{1}\left(t+t_{1}\right)>y_{2}\left(t+t_{2}\right)$. Hence, (4.2d) and (4.3d) cannot both be satisfied.

An immediate implication of Lemma 1 and inequality (4.1) is

Proposition 2. Prior to invention, the economy in which the substitute will, upon discovery, be controlled by a patent, is excessively conservationist:

$\tilde{p}(S)>p^{s}(S)$

for all $S$.

In Stiglitz \& Dasgupta, it was shown (Proposition $3 \mathrm{~b}$ ) that if the substitute were controlled by a monopolist, then the date of innovation, for any given 
initial stock of the resource at the date of invention, would be delayed. The actions of the (competitive) resource owners prior to invention thus exacerbate further this delay in the date of innovation.

\section{The Monopoly Arbitrage Equation}

The monopolist, in deciding his extraction policy, compares the marginal return from extracting now, to the expected marginal return from postponing. Let $V^{m}(S)$ be the present discounted value of a stock $S$ for a pure monopolist (i.e., a monopolist in both the resource and the substitute). Thus

$V^{m}(S) \equiv \max _{\left\{Q(t) . T^{m}\right\}}\left\{\int_{0}^{T^{m}} R(Q) e^{-r t} d t+e^{-r T^{m}} \max \frac{R(Q)-\bar{p} Q}{r}\right\}$ s.t. $\int_{0}^{T^{m m}} Q(t) d t \leqslant S$.

where

$$
\begin{aligned}
R(Q) & =p(Q) Q=\text { revenues when sales are } Q \\
T^{m} & =\text { date of exhaustion of the natural resource }
\end{aligned}
$$

$\max \frac{R(Q)-\bar{p} Q}{r}=$ the present discounted value of the profits generated by the

(We know from Stiglitz \& Dasgupta (forthcoming) Proposition 2a, that the optimal pattern of production and resource extraction for a pure monopolist always takes on the form represented by (5.1), i.e., a first phase in which only the resource is extracted; and a second phase in which only the substitute is produced.)

Let $M(S)$ represent the fall-back marginal revenue, the marginal return to having an extra unit of the resource at the date of invention, i.e.,

$\hat{M}(S) \equiv V^{m^{\prime}}(S)$

Thus, by reasoning identical to that employed in Section II, the expected present discounted value of postponing extraction from $t$ to $t+\theta$ is

$\lambda_{t} \theta \hat{M}\left(S_{t}\right)+\left(1-\lambda_{t} \theta\right)(M+d M)=\left(1+r_{t} \theta\right) M$

which on taking limits as $\theta \rightarrow 0$, yields the basic monopoly arbitrage condition

$\frac{\dot{M}_{t}}{M_{t}}=r_{t}+\lambda_{t}\left[1-\frac{\hat{M}\left(S_{t}\right)}{M_{t}}\right]$

With constant elasticity demand curves, this then can be written as

$$
\frac{\dot{p}}{p}=r_{t}+\lambda_{t}\left[1-\frac{\hat{M}\left(S_{t}\right)}{\varepsilon-1} p\right]
$$

Scand. J. of Economics 1981 
Thus, a comparison of the competitive and monopoly pre-invention rates of extraction is reduced to a comparison between

$\frac{M(S) \varepsilon}{\varepsilon-1}$ and $\hat{p}^{s}(S)$

For a pure monopolist, the marginal revenue from resource extraction is (in present discounted value terms) identical at all dates after this invention and equal to

$\hat{M}(S)=\hat{p}^{m}(S) \frac{\varepsilon-1}{\varepsilon}$

where $\hat{p}^{m}$ is the level to which the monopolist drops the price after the invention. Since we established in Stiglitz and Dasgupta (forthcoming) that (Proposition $2 \mathrm{~b}$ )

$\hat{p}^{m}(S)>p^{s}(S)$ for all $S$

it immediately follows (upon again using Lemma 1)

Proposition 3a. During the pre-invention phase the monopolist is unambiguously more conservation-minded than is socially optimal.

By the same token, from Lemma 1 and the fact that $\tilde{p}(S)<p^{m}(S)$ for all $S$ (Stiglitz \& Dasgupta, Proposition $3 \mathrm{~b}$ ), the pure monopoly price exceeds the price when the resource is competitively controlled but the substitute is controlled by a monopolist, we immediately observe

Proposition $3 \mathrm{~b}$. An economy in which the resource is competitively owned but the substitute is controlled by a patent is more conservation-minded than is socially optimal but less so than a pure monopolist.

It is this limited competition case which conforms to our intuition that mixed cases (limited competition) ought to lie between the polar cases of pure competition and pure monopoly.

\section{Resource Owned by a Monopolist, Substitute} Competitively Produced

The analysis of this case is almost identical to that of the preceding except that now we define

$V^{*} \equiv \max \int_{0}^{\infty} R(Q) e^{-r t} d t$

s.t. $p(t) \leqslant \bar{p}$

and

$\int_{0}^{\infty} Q(t) d t \leqslant S_{0}$ 
$V^{*}$ is the maximized present discounted value of the revenue generated by the resource when there is a competitive substitute available, which will be produced at the price $\bar{p}$. (The asterisk is simply used to denote this market structure.)

We can again show (with precisely the same line of argument) that, prior to the invention, the resource-monopolist will allocate his resources so that the. arbitrage equation (5.4) is satisfied; but now, $\widehat{M}(S)$, the marginal revenue of an extra unit of resource in the post-invention era, must be calculated in a different way. The marginal revenue is no longer necessarily proportional to price. In our earlier analysis, we showed that if $T_{2}$ denotes the date of final exhaustion of the natural resource, measured from the date of invention, then the marginal revenue at the date of invention is

$\bar{p} e^{-\tau T_{2}}$

and that this equalled $\hat{p}(\varepsilon-1) / \varepsilon$ if and only if the fall-back price, $\hat{p}<\bar{p}$, i.e. if and only if $S$ were large. The competitive supply of the substitute imposes a limit, $\bar{p}$, on the price which the monopolist of the resource can charge; extra units of the resource are sold at $\bar{p}$ at $T_{2}$.

To analyze prices prior to invention, we thus need to compare $\left(\hat{M}^{*}(S) \varepsilon\right) /(\varepsilon-1)$, marginal revenue in this regime, with $\left(\widehat{M}^{m}(S) \varepsilon\right) /(\varepsilon-1)$ under pure monopoly, on the one hand, and with $p^{s}$ and $\tilde{p}$ on the other. Proposition $4 \mathrm{~b}$ of Stiglitz \& Dasgupta established that

$\hat{M}^{m}(S)<\hat{M}^{*}(S)$

even when $\dot{p}^{m}>p^{*}(S)$, i.e. even when the monopoly price exceeded the price in this regime, marginal revenue was less, while Propositions $(2 \mathrm{~b})$ and $(3 \mathrm{~b})$ established that

$\frac{\hat{M}^{*}(\varepsilon-1)}{\varepsilon}>\hat{p}^{m}>p^{s}$

Using (6.2) and Lemma 1, we thus obtain

Proposition 4. $p^{*}(S)>p^{m}(S) \tilde{p}>(S)>p^{s}(S)$. If the substitute is supplied competitively while the resource itself is controlled by a monopolist, then pre-invention price is even higher than in pure monopoly, and hence innovation is delayed even more.

The intuitive interpretation of this result is that, by pursuing a more conservation-minded strategy, the resource owner is able to extend the period of his "monopoly" control of the market. This result is strengthened when the rate of technical progress is endogenous; see Dasgupta, Gilbert \& Stiglitz (1980b).

Scand. J. of Economics 1981 


\section{Duopoly}

The final case we consider is that where the resource and the substitute are controlled by two different monopolists. We then have, after the invention, a (potential) duopoly market. In our earlier study, we were able to characterize the Nash equilibrium as having three phases: in the first only the resource owner produced, and price was lower than $\bar{p}$; in the second both are produced, and market price is between $\bar{p}$ and $\bar{p}(\varepsilon /(\varepsilon-1))$; and in the final stage, $p=$ $\bar{p}(\varepsilon /(\varepsilon-1))$ and only the substitute is produced. We were able to obtain a differential equation describing the price movements in this duopoly model, and to show that $\hat{p}^{d}(S) \geq p^{m}(S)$ as $S \geq S^{*}$ : the duopoly fall-back price, $\hat{p}^{d}(S)$, was smaller or larger than the pure monopoly price as the stock at the date of invention was smaller or larger than some critical level $S^{*}$.

But just as in the previous case, the relationship between price and marginal revenue is complicated. In Stiglitz \& Dasgupta (forthcoming), we were able to show that

$\hat{M}^{d}(S)>M^{m}(S)$ for $S<S<S^{*}$ and $S \geqslant S^{*}$;

even though, for $S<S$, the duopoly price is higher, marginal revenue is lower. ${ }^{1}$ Indeed, since

$\hat{M}^{d}=p(1-\mu / \varepsilon)$

where $\mu$ is the fraction of the market supplied by the resource producer, for $S$ near $0, \mu \approx 0$, and hence

$\hat{M}^{d} \approx p=\frac{\bar{p} \varepsilon}{\varepsilon-1}$,

which may be considerably greater than $\widehat{M}^{m}$ :

$\bar{M}^{m} \approx \bar{p}$.

We can use these results, in conjunction with Lemma 1, to establish

Proposition 5. In the Nash-equilibrium duopoly, the resource owner in the preinvention era is more conservation minded than the pure monopolist and (a fortiori) than is socially optimal, provided the initial stock of the resource is not too large: prices, at least initially, with limited competition may be much higher than with pure monopoly.

\section{Concluding Remarks}

The basic results of this paper can be summarized in Fig. 6, giving the price of the resource as a function of the stock available, during the period prior to the invention having been discovered.

$\overline{1 \text { For } \hat{S}}<S<S^{*}$, we conjecture that (7.1) still holds, but we have not been able to prove it. $22-814811$

Scand. J. of Economics 1981 


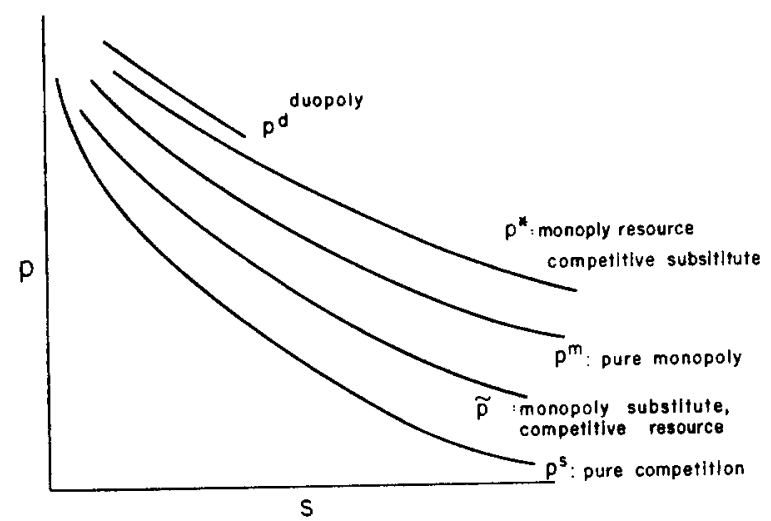

Fig. 6.

The result of particular interest relates to the competitive provision of the substitute: one of the strategies proposed for responding to the OPEC cartel is for the consuming nations to develop substitutes which they could then ensure would be competitively supplied. The response of a resource monopolist to this change in market structure would be to raise his prices, not to lower them. Although in the post-invention phase, his prices would be lower, his marginal revenue might be higher, and it is the effect on marginal revenue which is critical for determining his supply behavior in the pre-invention phase.

If one is concerned with using competition as a mechanism for lowering the price of a natural resource, but if one is unable to attain a perfectly competitive market, it appears to be critical precisely how competition is introduced: some forms of competition may serve to raise rather than to lower prices.

\section{References}

Arrow, K.: Economic welfare and the allocation of resources for inventors. In The rate and direction of inventive activity: Economic and social factors (NBER) (ed. R. Nelson). Princeton Univ. Press, 1962. Dasgupta, P., Gilbert, R. \& Stiglitz, J. E.: Invention and innovation under alternative market structures: The case of natural resources. Econometric Research Program Memorandum No. 263, Princeton, March 1980a.

Dasgupta, P., Gilbert, R. \& Stiglitz, J. E.: Energy resources and research and development. Erschöpfbare Resourcen. Duncker and Humbolt, Berlin, $1980 b$.
Dasgupta, P. \& Heal, G. M.: The optimal depletion of exhaustible resources. Review of economic Studies, Symposium on the Economics of Exhaustible Re. sources, pp. 3-28.

Dasgupta, P. \& Heal, G. M.: Economic theory and exhaustible resources. Chapter 11. (Cambridge Handbook), 1978.

Dasgupta, P. \& Stiglitz, J. E.: Industrial structure and the nature of innovative activity. Economic Journal, June 1980a (90), pp. 266-293.

Dasgupta, P. and Stiglitz, J. E.: Uneertainty, Industrial Structure and the Speed of R \& D, Bell Journal of Eco.

Scand. J. of Economics 1981 
nomics, Vol. 11, No. 1, Spring 1980b, pp. 1-28.

Dasgupta, P. \& Stiglitz, J. E.: Resource depletion under technological uncertainty. Econometrica, Jan. 1981, Vol. 49, pp. 85-104.

Herfindahl, O. C.: Depletion and economic theory. In Extractive resources and taxation (ed. M. Gaffney). University of Wisconsin Press, Madison, 1967.

Hotelling, H.: The economics of exhaustible resources. Journal of Political Economy $39,137-175$.

Solow, R.: Intergenerational equity and exhaustible resources. Review of Eico. nomic Studies, pp. 29-45, 1974a.

Solow, R. M.: The economics of resources or the resources of economics. American Economic Review, Papers and Proceodings, pp. 1-14. May $1974 b$.

Stiglitz, J. E.: Growth with exhaustible natural resources: Efficient and optimal growth paths. Review of Economic Studies, Symposium on the Economics of Exhaustible Resources, pp. 123-138, March 1974a.

Stiglitz, J. E.: Growth with exhaustible natural resources: Competitive growth paths. Review of Economic Studies, pp. 139-152, March $1974 b$.

Stiglitz, J. E.: Monopoly and the rate of extraction of exhaustible resources. American Economic Review, 1977.

Stiglitz, J. E. \& Dasgupta, P.: Market structure and resource depletion. Journal of Economic Theory, forthcoming.

Stiglitz, J. E., Gilbert, R. \& Dasgupta, P.: Invention and innovation under alternative market structures: The case of natural resources. Mimoographed, 1978.

Sweeney, J.: Economics of depletable resources: Market forces and intertemporal bias. Review of Economic Studies 44, 125-142, February 1974. 
Copyright of Scandinavian Journal of Economics is the property of Wiley-Blackwell and its content may not be copied or emailed to multiple sites or posted to a listserv without the copyright holder's express written permission. However, users may print, download, or email articles for individual use. 